\title{
Plasmodium knowlesi gene expression differs in ex vivo compared to in vitro blood-stage cultures
}

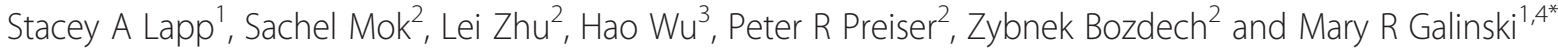

\begin{abstract}
Background: Plasmodium knowlesi is one of five Plasmodium species known to cause malaria in humans and can result in severe illness and death. While a zoonosis in humans, this simian malaria parasite species infects macaque monkeys and serves as an experimental model for in vivo, ex vivo and in vitro studies. It has underpinned malaria discoveries relating to host-pathogen interactions, the immune response and immune evasion strategies. This study investigated differences in P. knowlesi gene expression in samples from ex vivo and in vitro cultures.
\end{abstract}

Methods: Gene expression profiles were generated using microarrays to compare the stage-specific transcripts detected for a clone of $P$. knowlesi propagated in the blood of a rhesus macaque host and then grown in an ex-vivo culture, and the same clone adapted to long-term in vitro culture. Parasite samples covering one blood-stage cycle were analysed at four-hour intervals. CDNA was generated and hybridized to an oligoarray representing the $P$. knowlesi genome. Two replicate experiments were developed from in vitro cultures. Expression values were filtered, normalized, and analysed using $\mathrm{R}$ and Perl language and applied to a sine wave model to determine changes in equilibrium and amplitude. Differentially expressed genes from ex vivo and in vitro time points were detected using limma R/Bioconductor and gene set enrichment analysis (GSEA).

Results: Major differences were noted between the ex vivo and in vitro time courses in overall gene expression and the length of the cycle (25.5 hours ex vivo; 33.5 hours in vitro). GSEA of genes up-regulated ex vivo showed an enrichment of various genes including SICAvar, ribosomal- associated and histone acetylation pathway genes. In contrast, certain genes involved in metabolism and cell growth, such as porphobilinogen deaminase and tyrosine phosphatase, and one SICAvar gene, were significantly up-regulated in vitro.

Conclusions: This study demonstrates how gene expression in P. knowlesi blood-stage parasites can differ dramatically depending on whether the parasites are grown in vivo, with only one cycle of development ex vivo, or as an adapted isolate in long-term in vitro culture. These data bring emphasis to the importance of studying the parasite, its biology and disease manifestations in the context of the host.

Keywords: Plasmodium knowlesi, Malaria, Non-human primates, Macaca mulatta, Blood-stage infections, Microarrays, Antigenic variation, Gene expression, SICAvar, Haem

\section{Background}

Plasmodium invasion, growth and development in red blood cells (RBCs) is a complex process entailing many levels of controlled gene expression, receptor-ligand interactions, host cell modifications, and communication with the outside milieu. Each species of Plasmodium has

\footnotetext{
* Correspondence: mary.galinski@emory.edu

'Emory Vaccine Center, Yerkes National Primate Research Center, Emory University, Atlanta, GA, USA

${ }^{4}$ Department of Medicine, Division of Infectious Diseases, Emory University, Atlanta, GA, USA

Full list of author information is available at the end of the article
}

between 5,100 and 7,700 or so genes [1], which as first shown for Plasmodium falciparum, become differentially expressed throughout the parasite's intra-developmental cycle (IDC) in the blood [2-4]. Understanding the multitude of molecular and cell biological events required by Plasmodium to successfully parasitize RBCs and evade host defenses is paramount and increasingly within reach with post-genomic approaches [5-7].

The development of a continuous in vitro blood-stage culture system for P. falciparum was an essential step towards significantly advancing research in many of 
these areas of blood-stage malariology, as well as the development of malaria diagnostics, drug and vaccine candidate interventions [8]. A comparably robust bloodstage culture system is also available for the simian malaria species Plasmodium knowlesi $[9,10]$, but not other primate malaria species. Attempts have been made to develop continuous cultures of Plasmodium vivax [11] and the very closely related simian malaria parasite species Plasmodium cynomolgi [12], but the preference for reticulocyte host cells along with other possible requirements that have yet to be discovered have so far prohibited the development of routine long-term culture systems for these species [13-15].

While the utility of $P$. falciparum and P. knowlesi in vitro blood-stage culture systems has been evident for many avenues of research, including genetic manipulation to study gene expression characteristics and determine functional traits, the biological states of parasites in host organisms can differ substantially from that of in vitro cultured parasites. For example, this became evident for P. falciparum with recognition of the loss of non-essential gene segments in culture [16] and reviewed in [17]. Expression of the variant antigen var gene transcripts from $P$. falciparum have also been shown to become down-regulated when clinical isolates are adapted to in vitro culture $[18,19]$. Numerous changing metabolic and physical parameters in the parasite and host factors are either different or lacking in vitro, which may affect the parasite's normal growth and biology. Investigations have shown that even one altered parameter, such as glucose level, can result in a marked change in gene expression profiles in cultured P. falciparum, with 560 genes exhibiting a two-fold or greater change in the presence of $0.6 \mathrm{mM}$ glucose compared to $11.1 \mathrm{mM}$ glucose [20].

Notably, P. knowlesi can readily be studied in vivo, ex vivo and in vitro, and it can potentially serve as a model for $P$. falciparum or $P$. vivax; depending on the scientific question. Plasmodium knowlesi is currently also recognized as a species that can be transmitted to and cause illness and death in humans [21-23]. Given its broad utility, many critical basic malaria research discoveries have been made using $P$. knowlesi. These include classic investigations of merozoite attachment to and invasion of erythrocytes after the formation of a tight junction $[24,25]$, the first report of the phenomenon of antigenic variation (in rhesus monkeys) [26], and the first identification of parasite-encoded variant antigens expressed at the surface of infected RBCs; known as the Schizont Infected Cell Agglutination (SICA) antigens [27]. The SICA antigens are encoded by a large multigene family known as SICAvar [28-30], which is functionally analogous and evolutionarily related to the var gene family in P. falciparum [31,32]; both gene families are highly expressed in ring stages $[28,30,33]$. Expression of the SICA proteins in vivo in rhesus monkeys is associated with the increased virulence of $P$. knowlesi, and both the expression of these proteins and switching of variant types at the surface of the infected host cells is dependent on the presence of the spleen [34]. Recent studies demonstrate for the first time that SICA[-] parasites lack full-length SICAvar transcripts and lack SICA protein, consistent with apparent post-transcriptional processing and translational repression [30].

Particularly now with the expanding potential for postgenomic exploration of Plasmodium biology and pathogenesis, and the use of comprehensive systems biology approaches to understand host-pathogen interactions, it is important to define and be cognizant of the similarities and differences in gene expression that may occur when Plasmodium is grown in a living host, compared to in vitro. Here, these parameters are examined by studying the stage-specific gene expression profiles of $P$. knowlesi blood-stage parasites when grown in a rhesus monkey followed by one cycle of ex vivo growth and development compared to long-term in vitro culture. The majority of the observed microarray signals correspond to genes that are annotated in the current $P$. knowlesi genome database as hypothetical. Of the various differentially expressed (DE) known genes, it is striking that signals representing a large number of SICAvar gene transcripts were strongly up-regulated in the ex vivo (ring-stage) samples, but not in the in vitro samples. These observations in toto are germane for understanding the biology of the $P$. knowlesi intraerythrocytic development cycle (IDC) and the pathogenesis and virulence of this species. The results are also highly pertinent as indicators of what biological or biochemical networks and pathways may likewise be affected when other species including P. falciparum are grown and propagated long-term under in vitro conditions.

\section{Methods}

\section{Ethics statement}

Animal experimentation reported here involved one animal (Macaca mulatta; rhesus macaque) and was conducted in AAALAC-accredited facilities at the Yerkes National Primate Research Center of Emory University in accordance with the Animal Welfare Act and the Guide for the Care and Use of Laboratory Animals. Emory University's Institutional Animal Care and Use Committee (IACUC) approved this project's experimental protocol. Temperatures in animal areas are controlled and monitored by an automated on-line system. The system has set parameters for high and low temperatures, maintained at $22^{\circ} \mathrm{C} \pm 3^{\circ}$. The photoperiod is set at a $12 / 12$ cycle. Rhesus macaques are housed singly, and provided with one of the following: forage board, peanut feeder, challenger ball, or other similar device for 
enrichment. In addition, destructible enrichment is distributed as prescribed to monkeys. Rhesus macaques receive jumbo biscuits (15\% protein). Animals are fed twice daily. The amount of food is adjusted depending on species, sex, age, weight and specific number of animals living in a group. Consumption is monitored and adjustments are made as necessary so that animals get enough food with minimum waste. Enrichment produce is fed twice weekly. All animals are monitored daily by veterinary staff for potential health problems. Experienced personnel trained all animals to voluntary present at the front of the cage for skin prick using positive reinforcement. For blood collection, as required by this study, animals are sedated with Ketamine $(10 \mathrm{mg} / \mathrm{kg}$ $\mathrm{IM})$, which is allowed no more than twice per 24 hours. Per IACUC guidelines, maximum blood volume collected cannot exceed $10 \mathrm{ml} / \mathrm{kg} / \mathrm{month}$. The one rhesus macaque assigned to this study was treated and available for other studies at the Yerkes National Primate Research Center.

\section{Parasite samples for microarray analysis}

An ex vivo P. knowlesi blood-stage culture was initiated using iRBCs from a rhesus macaque infected with the Pk1(A+) clone of P. knowlesi [27]. The Pk1(A+) clone was selected since the genomic DNA from this clone was used to develop the first reported $P$. knowlesi genome sequence [35]. Briefly, a cryopreserved vial of ringstage parasites was thawed, processed and inoculated into a rhesus monkey using standardized procedures. Parasitaemia was monitored daily and whole blood was collected into heparin-treated tubes when it reached $8 \%$ ring-stage infected RBCs (iRBCs). The monkey was treated with $15 \mathrm{mg} / \mathrm{kg}$ of chloroquine for 3 days to clear the parasites. Collected whole blood was treated with a final concentration of $2 \mathrm{mg} / \mathrm{ml}$ ADP (adenosine diphosphate bis salt) for $5 \mathrm{~min}$ before passage through a glass bead column equilibrated with Hank's balanced salt solution to remove platelets, followed by passage through a Plasmodipur filter. The filtered blood was centrifuged for $5 \mathrm{~min}$ at $800 \mathrm{~g}$ and the pellet resuspended in RPMI with L-glutamine, supplemented with $0.25 \%$ sodium bicarbonate, $50 \mathrm{ug} / \mathrm{ml}$ hypoxanthine, $7.2 \mathrm{mg} / \mathrm{ml}$ HEPES, $2 \mathrm{mg} / \mathrm{ml}$ glucose, $10 \%$ human $\mathrm{AB}+$ serum. The iRBCs were cultured at $1 \times 10^{7} \mathrm{iRBCs} / \mathrm{ml}$ in multiple $175 \mathrm{~cm}^{2}$ flasks (one per time point, TP) and gassed with 5\%: $5 \%$ : 90\%, O2:CO2:N2. One culture flask was processed every four hours over a 24-hour period for extraction of parasite RNA, and these samples were designated as ex vivo TP \#s 1-6.

For the in vitro time course samples, a cryopreserved vial of culture-adapted $\mathrm{Pk} 1(\mathrm{~A}+)$ parasites was thawed using standardized procedures, and expanded in RPMI with L-glutamine, supplemented with $0.25 \%$ sodium bicarbonate, $50 \mathrm{ug} / \mathrm{ml}$ hypoxanthine, $7.2 \mathrm{mg} / \mathrm{ml}$ HEPES, $2 \mathrm{mg} / \mathrm{ml}$ glucose, 10\% human $\mathrm{AB}+$ serum. Fresh rhesus RBCs were added as necessary, and the parasitaemia was maintained at less than $10 \%$. The culture was synchronized by consecutive Percoll (52\%) and sorbitol treatment in which RBCs were suspended in $5 \mathrm{X}$ volume of pre-warmed $5 \%$ sorbitol, incubated for five minutes and washed three times in RPMI [36]. Since the in vitro cultured parasites develop at a slightly slower rate than in vivo, a 28- or 32-hour time course plan was designed for RNA extraction, with iRBC collection every four hours. Samples were generated representing two biological replicates (I and II), with samples for seven TPs collected for the first replicate and eight for the second; the eighth TP was particularly aimed at collecting very mature segmenting schizonts.

\section{RNA extraction}

For the first two time points from the ex vivo culture, $1 \times 10^{10}$ iRBCs were collected and put into Trizol LS (Invitrogen) at a 1:10 pellet to reagent ratio, vigorously shaken for $1 \mathrm{~min}$, incubated at $37^{\circ} \mathrm{C}$ for $5 \mathrm{~min}$, and stored at $-80^{\circ} \mathrm{C}$. For all other time points from the ex vivo culture, $5 \times 10^{9}$ iRBCs were collected. At the last time point, a 1:20 pellet to Trizol LS ratio was used to extract RNA. All samples were immediately stored at $-80^{\circ} \mathrm{C}$. For the in vitro cultures, approximately $5 \times 10^{9}$ parasites were collected for the first two time points, and $1 \times 10^{9}$ parasites were collected for the remaining time points. To purify the total RNA, the parasite/Trizol samples were thawed at room temperature and transferred to $30 \mathrm{ml}$ Teflon tubes. One $\mathrm{ml}$ chloroform per $5 \mathrm{ml}$ sample volume was added and vigorously shaken for $1 \mathrm{~min}$ before centrifuging at $12,000 \mathrm{~g}$ for $30 \mathrm{~min}$ at $4^{\circ} \mathrm{C}$. The aqueous layers were removed to a $30 \mathrm{ml}$ polypropylene tube, 0.8 volume of isopropanol added, and incubated overnight or for at least 2 hours at $-20^{\circ} \mathrm{C}$ before centrifuging at $12,000 \mathrm{~g}$ for $30 \mathrm{~min}$ at $4^{\circ} \mathrm{C}$. The supernatant was carefully removed and the pellet was allowed to dry for 5-10 $\mathrm{min}$ at room temperature and then resuspended in $1 \mathrm{ml}$ of RNase-free water. The sample was purified using the RNeasy midi kit (Qiagen) following the manufacturer's instructions.

\section{Proteomic analysis}

Briefly, late stage trophozoites from synchronized cultures were purified with $52 \%$ percoll, washed 3 times with incomplete RPMI 1640, resuspended in SDS sample buffer and stored at $-80^{\circ} \mathrm{C}$. Immunoprecipitation with a pan-SICA antibody was performed as in [30]. Peptides were generated as previously described [31]. Protein bands were excised from SDS-PAGE gels and gel pieces destained and dried. Dried gel bands were digested at 
$37^{\circ} \mathrm{C}$ overnight with $0.4 \mathrm{~g}$ of proteomics grade trypsin (Sigma, St. Louis, MO, USA). The resulting peptides were then extracted with $0.1 \%$ trifluoroacetic acid in $50 \%$ acetonitrile (Sigma), desalted and concentrated using ZipTip pipette tips containing $\mathrm{C} 18$ reversed-phase media (Millipore, Billerica, MA), and then washed in $0.1 \%$ trifluoroacetic acid and eluted in $0.1 \%$ trifluoroacetic acid $/ 50 \%$ acetonitrile (Sigma). Cleaned peptides were analysed by reverse-phase LC-MS/MS using an LTQOrbitrap mass spectrometer (Thermo Finnigan). A reverse database strategy using the SEQUEST algorithm was implemented to evaluate false discovery rate; the matched peptides were filtered according to matching scores to remove all false matches from the reverse database [37]. Only proteins that were matched by at least two peptides were accepted to further improve the confidence of identification. The peptides were then searched against the NCBI database, with searches being limited to Plasmodium results. The $P$. knowlesi identified protein sequences were then used to BLAST (Basic Local Alignment Search Tool) against the NCBI database for sequence homologies.

\section{Data analysis}

\section{Reannotation of oligonucleotide probes}

A set of 60-mer oligonucleotide probes (Additional file 1) developed prior to the publication of the P. knowlesi genome was reannotated based on the genome sequence reported in PlasmoDB V8.2. All the oligonucleotides in the array were BLASTed against the PlasmoDB V8.2 sequences using NCBI BLAST 2.2 with the parameter of E-value cutoff at 0.1 . Only oligonucleotides with unique hits to protein-coding regions were kept for further analysis. The unique hit oligonucleotides were defined as probes having high first-hit bit scores $(>85)$ and low second-hit bit scores $(<60)$ or probes having second-hit bit scores equal to 0 . Of the 4,799 P. knowlesi probes found to have unique hits, 4,755 representing 4,700 protein-coding genes were used in the analysis.

\section{Determining the periodicity of gene expression Microarray hybridization, data filtering and normalization} All data were processed using $\mathrm{R}$ and Perl language. The workflow is shown in Additional file 2. cDNA synthesis, Cy dye labelling, as well as microarray hybridizations of the time course samples were carried out as described previously [38]. For each P. knowlesi time course, sampling time point RNAs labelled with Cy5 were hybridized against a reference RNA pool labelled with $\mathrm{Cy} 3$ (consisting of equal amounts of $P$. knowlesi RNA from each time point). Microarray hybridizations were carried out on a chip with 4,992 60-mer oligonucleotides representing 4,700 coding genes designed for $P$. knowlesi, with the remainder representing $P$. vivax genes. Microarray data acquired with GenePix Pro 6.0 software (Axon Instruments, USA) were filtered for quality control by accepting only features with $\mathrm{Cy} 3$ and $\mathrm{Cy} 5$ signal intensities higher than two-fold of the microarray background intensities. The $\log 2$ ratio of $\mathrm{Cy} 5 / \mathrm{Cy} 3$ intensity was calculated after background subtraction and was normalized using locally estimated scatterplot smoothing (LOESS) in marray package of $\mathrm{R}$. To reduce technical noise between experiments, robust quantile normalization was applied to the data across all time points to achieve the same distribution of $\log 2$ ratios (Affy package of $\mathrm{R}$ ). After normalization, probes were filtered out that had more than two missing values across all time points for any one of the three time courses, resulting in 4,198 probes, representing 4,165 protein-coding genes that were used for further analysis (Additional file 3). The microarray probe ratios were averaged for multiple probes of a particular gene. Data for the microarrays and probe information are available at the Gene Expression Omnibus database at the National Center for Biotechnology Information [GEO: GSE62886, GPL19374].

\section{Sine wave modelling}

To compare gene transcription between the iRBC samples without knowing the exact time post-invasion, a sine wave model was applied. The function is described in Figure 1d. $t$ is the time (hours) at each time point, and $y(t)$ is the corresponding transcription levels at each time point. Equilibrium is the average level of one complete sine curve. It was assumed to be the balanced transcription level of a gene across the life cycle, an original state that a gene would return after expression peak, valley and any expression changes. Amplitude is the transcription level change according to equilibrium, visually represented as the vertical distance between the extrema of the sine curve and the equilibrium value. $h$ is the period of one complete life cycle expressed in hours and phase is the fraction of one life cycle indicating the time offset of an expression peak to the time point at one quarter of the life cycle. For each gene, the parameters equilibrium, amplitude, and phase were determined and $h$ optimized within 12 to 48 by the sine wave model to find a sine function that best fits the periodical pattern of gene transcription levels across the life cycle.

\section{Phase adjustment}

Phase is the initial angle of a sinusoidal function at its origin. It indicates the time offset of an expression peak to time zero. The magnitude of the phase correlates to the timing of the peak expression of a gene. Since it is difficult to synchronize cells at the exact same starting time, the calculated phase for the same gene from time course to time course would differ. Therefore, phase adjustment is required to adjust for these differences. 


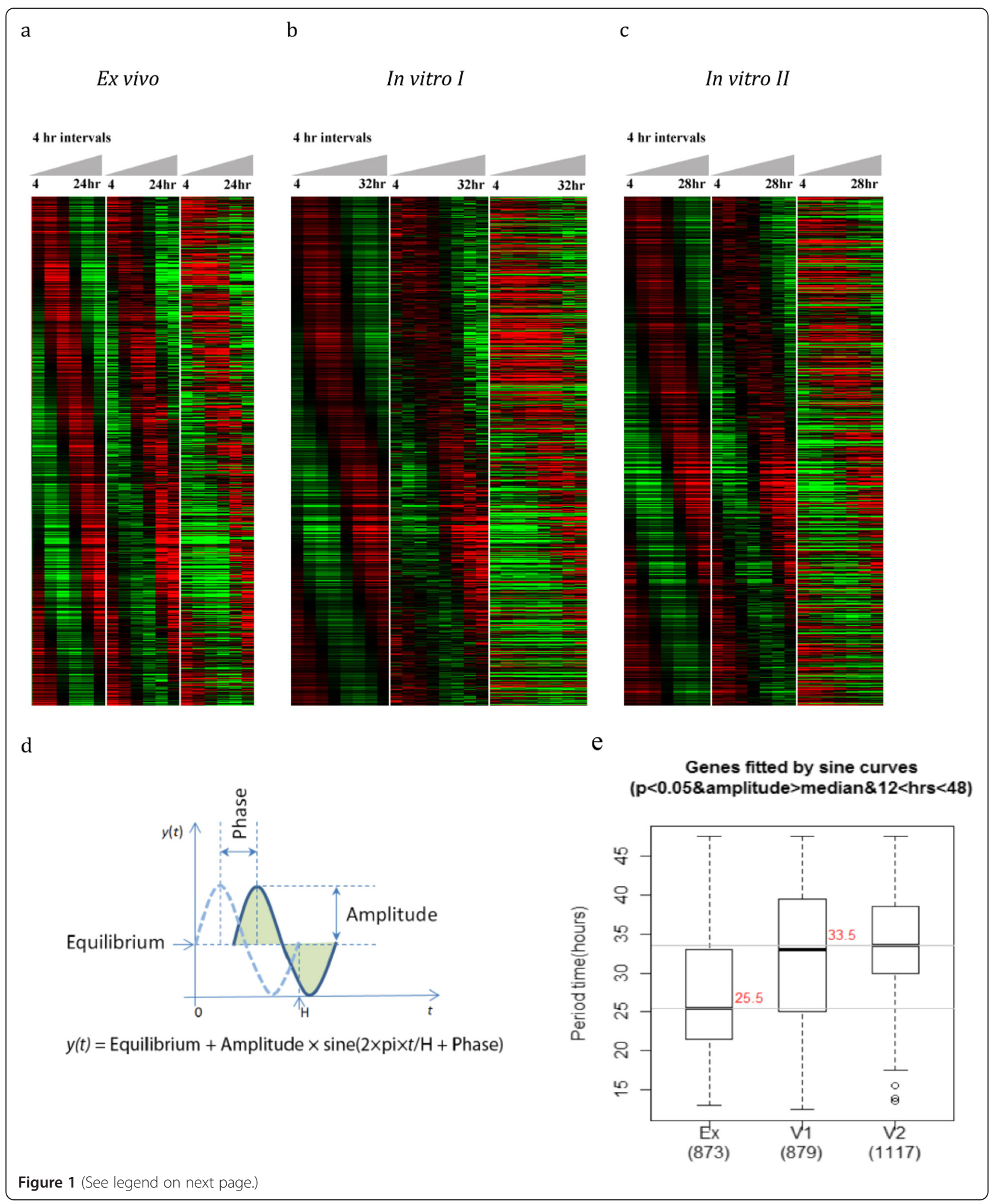


(See figure on previous page.)

Figure 1 Periodical transcription of $P$. knowlesi genes during the IDC. Transcriptomes showing 4,165 PK genes of the ex-vivo (a) and two in-vitro samples $(\mathbf{b}, \mathbf{c})$ of the time course sampled at 4 hour intervals over 24-32 hours in culture. First column represents raw data during time courses; second column represents raw data after median centered normalization and third column represents equilibrium value centered data after smoothing by sine functions with $\mathrm{H}=27$ for ex vivo and $\mathrm{H}=33$ for in vitro. (d) Sine wave model and its equation. $t$ is the time (hours) at each time points, and $y(t)$ is the corresponding transcription levels at each time point. Equilibrium is the average level of one complete sine curve. Amplitude is visually represented as the vertical distance between the extrema of the sine curve and the equilibrium value. $h$ is the period of one complete life cycle expressed in hours and phase is the fraction of one life cycle indicating time offset of an expression peak to the time point at one quarter of life cycle. (e) Prediction of duration for a complete cycle of ex vivo and in vivo samples (period time). $873 \mathrm{genes}$ from ex vivo sample, 879 and 1117 genes from in vivo samples I and II significantly fit a sine curve ( $p$ value $<0.05$ and amplitude above median) and were used to predict the period. The median period is 25.5 hours for ex vivo sample, and 33.5 hours for in vitro samples.

Using the established sine wave model, expression levels were estimated for each gene at each hour during its life cycle to give rise to 25 time points (ex vivo) and 33 time points (in vitro). The in vitro time points were mapped to ex vivo time points by determining the highest correlated time point between ex vivo and in vitro expression profiles (Additional file 4). To make the result more reliable, only 219 genes having significant periodical expression patterns were used ( $p$ value $<0.05$ and amplitude $>0.3$ of their sine curve) in all three samples. Phase shift was estimated for each in vitro time point based on the corresponding ex vivo sample. The average of phase shift then was used as the delta phase, 0.85 between in vitro I and ex vivo, 1.4 between the biological replicate in vitro II and ex vivo.

\section{Functional enrichment analysis}

Gene set enrichment analysis (GSEA) was carried out based on GO terms, KEGG pathway and MPM pathway annotated for each $P$. knowlesi gene according to their $P$. falciparum orthologs (PlasmoDB v8.2) [39]. 4,570 of 5,197 protein-coding genes in P. knowlesi have orthologs in P. falciparum $(3,838 P$. knowlesi genes with at least one GO term, 761 genes with at least one KEGG identification, and 2,346 genes with at least one MPM identification). In the gene enrichment analysis, only functional groups with more than 10 genes were considered. In addition, another eight functional groups were added including SICAvar, kir and others derived from recent work [40].

\section{Differential expression analysis by time point}

For each microarray, logarithms of fold changes between the various experimental time point sample RNAs and reference RNAs were first computed for all genes. Then cross-array normalization was applied to remove global technical biases brought by different arrays. For each array the log-fold changes were shifted and rescaled so that they have the same means and variances. There are expression values for each gene at six time points for the ex vivo time course and seven time points for the two in vitro time courses. To make the data comparable for different samples, the seven expression values for the in vitro samples were mapped into six time points by interpolation (Additional file 5).

\section{Assessing global differences in gene expression among the three time courses}

To assess the global differences in gene expression among the ex vivo and in vitro samples, the pair-wise differences of expression were computed among the three samples for all genes. For a gene, the difference score between two samples is defined as the Euclidean distances for its expression at all time points. Smaller distance means greater similarity. By doing so three difference scores were obtained for each gene, whereby each represents the difference between a pair of samples. For a gene, if the difference score between the two in vitro samples is the smallest among the three, it indicates that the expression of that gene is more similar between the in vitro samples.

To rigorously assess the significance of this proportion, a randomization test was performed. In each permutation, gene labels were randomly shuffled for all samples but the time points were kept unchanged. Then the pairwise difference scores were recomputed for all genes, and the number of genes with difference scores between the in vitro samples being the smallest was computed.

\section{Detecting individual differentially expressed (DE) genes}

To detect individually DE genes from ex vivo and in vitro samples, the two sets of in vitro samples were treated as biological replicates. DE genes at each time point were detected using limma R/Bioconductor software package [41]. To detect $\mathrm{DE}$ genes considering data from all time points, a functional $t$-test procedure was adopted [42]. Specifically, for each gene a modified $t$ statistic was computed at each time point, and then the maximum of the t-statistics from all time points was obtained as the test statistics for the gene. The critical value for differential expression can be obtained through a permutation test. Because of the small sample size, stringent criteria were used and the maximum of all $t$-values from all permutations were used as the threshold for defining DE genes. 


\section{Results and discussion}

Microarray experiments were performed to evaluate the global gene expression profiles of $P$. knowlesi bloodstage parasites as they mature in host red blood cells, from ring to trophozoite and schizont stages of development, and compare the profiles between ex vivo cultures and in vitro adapted cultures. An overarching purpose of this research was to determine which gene expression patterns if any may be dependent on the presence of host factors.

A P. knowlesi infection was established in a naïve rhesus monkey and the maximum blood volume allowed by IACUC guidelines and approved protocols $(10 \mathrm{ml} / \mathrm{kg} /$ month) was drawn to obtain ring-stage iRBCs for ex vivo culture and the collection of stage-specific samples every 4 hours over the parasite's 24-hour IDC. Plasmodium knowlesi asexual stage development is synchronous in vivo, making this readily feasible, beginning with ringstage iRBCs collected from a blood draw. It would have been ideal from a scientific standpoint to obtain blood collections throughout the IDC, but, per IACUC guidelines, animals may only be sedated twice/day, and in addition to logistical limitations of the availability of animal resource staff over a 24-hr period, ex vivo cultures were by necessity initiated when the iRBCs were at the ring stage of development.

For comparison, two biological replicates were generated from in vitro adapted cultures, which do not maintain synchronous growth [9]. Acquiring these samples for comparison with the ex vivo cultures, therefore, required synchronization steps (see Methods). The first in vitro time course was collected over 28 hours. For the second in vitro time course an additional sample TP was collected at 32 hours to obtain segmented schizonts, the final stage of the parasite's growth before the release of merozoites. These cultures were maintained by the addition of freshly acquired RBCs from a different rhesus macaque than the one used for the ex vivo time course; in our view the specific host RBCs themselves would have negligible impact on the transcription profiles over one cycle compared to the culture environment (ex vivo versus long term in vitro). Notably, the $\mathrm{Pk} 1(\mathrm{~A}+)$ line is routinely grown in culture medium supplemented with human $\mathrm{AB}+$ serum. An overarching goal of this work is to compare parasite biology when adapted to widely available long-term culture conditions versus ex vivo. For this reason and consistency through multiple experiments, human $\mathrm{AB}+$ serum pooled from 10 different donors was used in lieu of rhesus macaque serum.

\section{Periodicity of gene expression in the $P$. knowlesi IDCs}

To test for periodicity of gene expression in the P. knowlesi IDCs, sine wave regression was carried out for each expressed gene during the established time courses. $3,684(\sim 88.5 \%)$ out of the 4,165 genes examined on the array significantly fit to a sine curve with a $\mathrm{p}$ value $<0.05$ (F test) for at least one of the time courses. These analyses confirm that a majority of the parasite's genes are transcribed periodically during the blood-stage cycle (Figure 1a, b and c), and each gene's transcriptional profile can be fit to a sine wave with a determined equilibrium, amplitude and frequency (Figure 1d). For each time-course experiment, the time required for the parasite to complete the blood-stage cycle of growth and development was estimated. For each gene, varying the cycle length from 12 hours to 48 hours identified the best-fit sine curve, and the optimized sine function with the minimum $\mathrm{F}$ test $\mathrm{p}$-value was gained. Based on this assumption, the length of each blood-stage cycle was estimated from genes that were significantly fitted by a sine curve ( $\mathrm{p}$ value $<0.05$ ) and amplitude above the median (based on 873 genes for the ex vivo time course, and 879 and 1,117 genes for the in vitro I and II time courses, respectively).

Parasites grown under in vitro conditions developed at a significantly slower rate ( 25 hours ex vivo versus 33 hours in vitro) (p value $<2.2 \mathrm{e}-16)$ (Figure 1e). Sine wave models were therefore established separately with the periodic time as 25 hours and 33 hours for the ex vivo and in vitro samples, respectively. Smoothed transcription profiles are shown in the second columns of the heat maps in Figure 1a, b and c. In addition, the age of the parasite at the start of the TP collections (TP1) was markedly different for each time course when the pair-wise correlation of TP1 was calculated between the time courses. This is a reflection of the inherent difficulty in precisely matching the ages of ring-iRBCs acquired directly from a rhesus macaque infection with those from synchronized in vitro cultures. Hence, data processing and adjustments were necessary to compare the ex vivo and in vitro transcriptomes.

\section{Comparative overview of the transcriptomes}

Using the sine wave model, DE genes between the transcriptomes were defined based on equilibrium, amplitude and phase parameters as calculated for each gene. Distinctive gene expression differences were not noted between the two in vitro transcriptomes by these methods, or others, and pair-wise differences of gene expression among the three time courses (ex vivo, and the two in vitro biological replicates) indicate that $77 \%$ of the expressed genes are in fact more similar between the in vitro time courses (Figure 2a). In a randomization test based on 1,000 permutations, the permutation p-value was determined to be less than 0.001 . These results strongly indicate that the gene expression profiles derived from the two in vitro time courses are much more similar 
(a) Difference scores

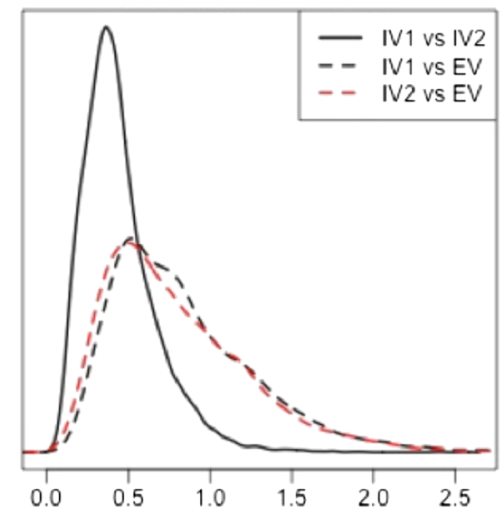

(b) Difference scores - permutation

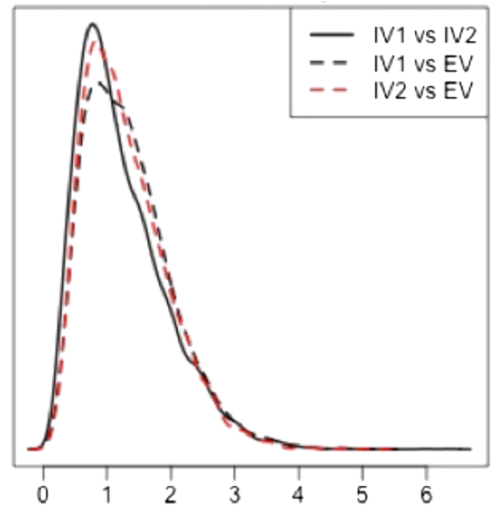

(c)
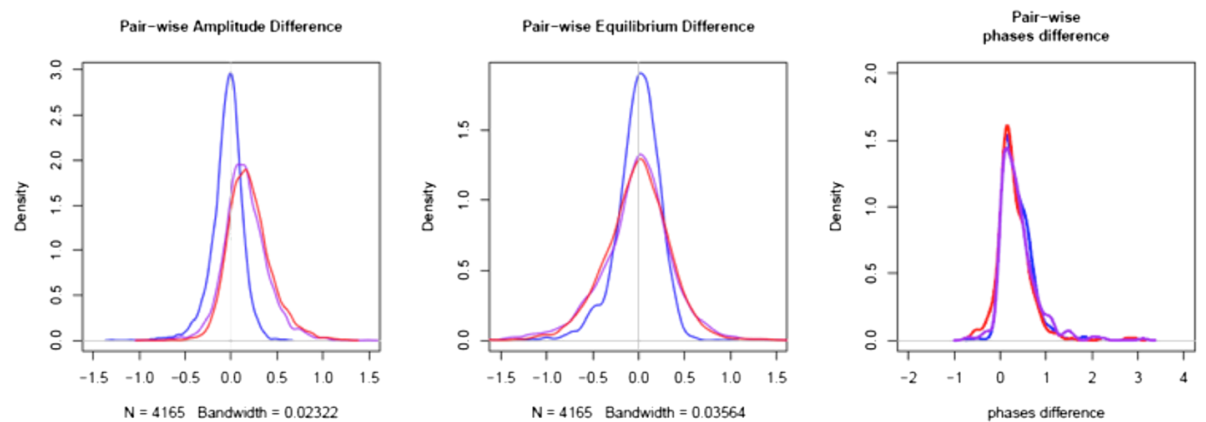

Figure 2 Overall differences between in vitro and ex vivo time courses. (a) The distributions of pair-wise difference scores for all genes showing the similarity of the two in vitro experiments and how they differ from the ex vivo experiment (b) the distributions of pair-wise difference scores from a permutation showing that the difference scores do not occur by chance. (c) Comparison of amplitude, equilibrium and phase between ex vivo and in vitro samples. These plots show the distribution of pair-wise difference of amplitude, equilibrium and phase values of ex vivo and in vitro I (red), ex vivo and in vitro II (pink), and in vitro I and in vitro II (blue). The amplitude difference was defined between ex vivo

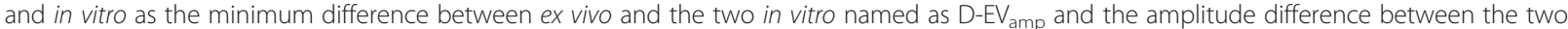
in vitro samples as D-W $W_{a m p}$. The equilibrium difference (D-EV eqm and D- $\left.W_{\text {eqmı }}\right)$ and phase difference $\left(D-E V_{p h}\right.$ and $\left.D-W_{\text {ph }}\right)$ were similarly defined.

to each other compared to the gene expression data from the ex vivo samples (Figure $2 \mathrm{~b}$ ). The pairwise differences in amplitude ( $\mathrm{p}$ value $<2.2 \mathrm{e}-16$ ) and equilibrium ( $\mathrm{p}$ value $<2.2 \mathrm{e}-16)$ are significantly smaller between in vitro samples than between in vitro and ex vivo samples. In addition, the amplitudes of gene expression were found to be significantly higher for the ex vivo TPs than the in vitro TPs ( $\mathrm{p}$ value $<2.2 \mathrm{e}-16$ ), which is likely a result of the greater degree of synchronicity of the ex vivo culture. In the phase analyses, as expected, no significant changes were observed among the ex vivo and in vitro samples (Figure 2c).

\section{Differentially expressed genes in equilibrium and amplitude}

Differentially expressed genes were defined in equilibrium and amplitude based on the criterion that the minimum differences among the ex vivo and each of the two in vitro cycles were greater than the differences noted from the in vitro replicates. Taking all 4,165 considered genes into account, 2,009 transcripts were identified with changes in equilibrium and 2,355 transcripts with changes in amplitude. The distributions of gene numbers at each fold change are shown in Figure 3. GSEA was performed as described using P. knowlesi orthologs of $P$. falciparum genes belonging to annotated functional gene sets/pathways from KEGG, GO and MPM databases [43-45], in addition to several P. knowlesi specific gene sets including the predominant large SICAvar and kir multigene families.

Importantly, among the 1,029 ( 51\%) genes that were up-regulated in equilibrium in the ex vivo samples, there was significant enrichment in genes coding for SICA proteins $(F D R=0)$. Other genes noted in this category are associated with biological processes relating to RNA transport, translation initiation, and phagosomes. Conversely, 980 ( $49 \%)$ of the genes that were down-regulated in equilibrium showed enrichment in transcripts involved in multiple biochemical pathways associated with nucleosome and chromatin assembly and organization, as well as 
(a)

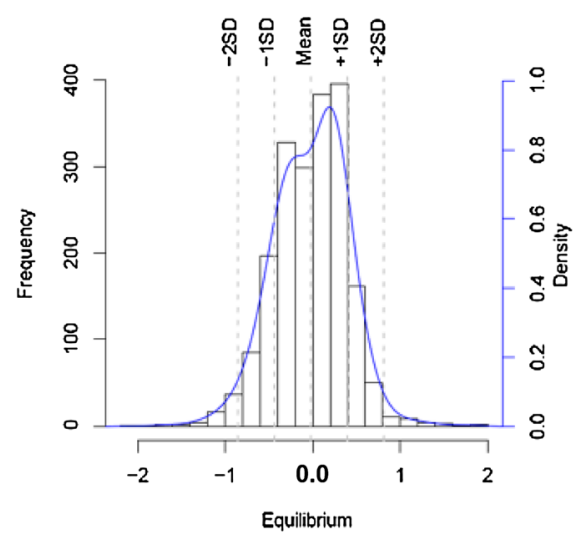

(b)

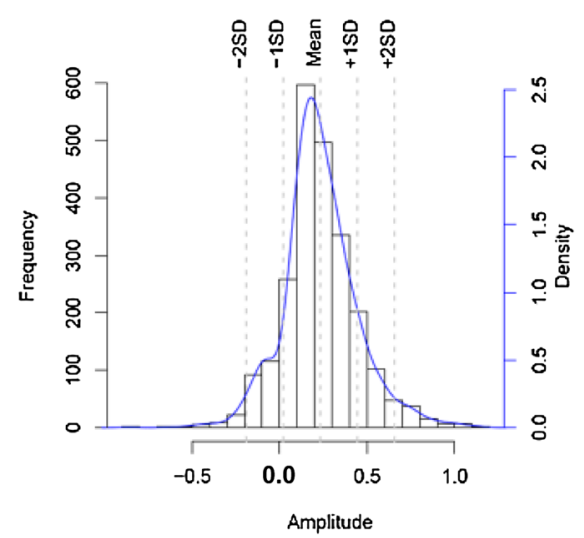

Figure 3 Histogram showing the frequency of DE genes in terms of fold change between ex vivo and in vitro samples with respect to amplitude and equilibrium ( $\log _{2}$ scale). (a) Number of genes differentially expressed in equilibrium at each fold change cutoff (b) Number of genes differentially expressed in amplitude at each fold change cutoff. The majority ( 89\%) of genes were up regulated.

DNA replication, proteasome-mediated proteolysis of proteins, and Maurer's cleft related proteins (FDR $<25 \%$, Figure 4a). Genes associated with ribosomal structure and fatty acid synthesis and certain organellar specific proteins (plastid, mitochondrion and ER) are among those that were less so, but also noticeably down-regulated in the ex vivo culture.

A majority $(2,108$ or $\sim 89 \%)$ of genes with changes in amplitude were up-regulated in the ex vivo cultured parasites. These genes mainly include ribosomal associated genes, SICAvar genes, Maurer's Cleft related protein genes and histone acetylation pathway genes, while the down-regulated genes $(247, \sim 11 \%)$ showed an enrichment to just a few invasion-related genes (FDR $<25 \%$, Figure 4b). As a whole, the results reveal that the ex vivo parasite has an overall higher capacity for transcribing certain genes involved in the interaction of hostparasites with larger stage-specific amplitudes, an effect apparently resulting directly from the in vivo growth of the parasite in rhesus monkeys, and in support of predictions to this effect. The up-regulated genes are accompanied by lowered transcription of various metabolic pathway genes such as for DNA replication, protein degradation and chromatin organization.

Significant DE genes were defined by applying the criterion that the minimum difference between the ex vivo and each of the in vitro cycles was greater than 1.5-fold (equal to Mean $\pm \sim 1.5 \mathrm{SD}$ ) of the difference between the in vitro replicates. 95 and 130 genes were up-regulated, and 162 and two genes were down-regulated, in equilibrium and amplitude, respectively.

\section{Differentially expressed genes by stage specificity}

To determine if the DE genes were biased towards any particular stage in the IDC, the transcription profiles of all DE genes were averaged, as shown in Figure 5a, b and c. Genes that were up-regulated in equilibrium in the ex vivo culture were most highly expressed in the early ring stage and late stage schizonts (Figure 5a), while genes that were down-regulated in the ex vivo culture had peak expression in the trophozoite and early (2-4 nuclei) schizont stages (Figure 5b). Similarly, genes that were up-regulated in amplitude in the ex vivo culture were also highly expressed in the trophozoite stage (Figure 5c). The major significantly enriched functional pathways for the DE genes had peak expression that coincided with the stage of peak expression observed here.

Taking all TPs into consideration using an alternative method that assesses calculated pairwise differences, 650 genes were found to be DE (FDR adjusted p-value $<0.01$ ) between the ex vivo and in vitro samples. The majority of these genes $(403 ; 62 \%)$ were annotated in the P. knowlesi genome database ( $\mathrm{Pk} 4$ assembly) as hypothetical genes $[1,35]$. Of note, sequences representing 32 SICAvar gene IDs were shown to be differentially up-regulated at the ring stage, which corresponds to $38 \%$ of the 101 SICAvar gene oligonucleotides present on the array (Figure 6). These 101 oligonucleotides represent 99 unique SICAvar gene IDs plus two contigs with SICAvar gene sequences with most annotated at the time of this study as gene fragments [35]. Some have since been confirmed as contiguous sequences that are part of the same gene [30]; others occur next to gaps in the assembly [35]. Thus, the SICAvar oligonucleotides on the microarray do not represent the entire reported SICAvar gene family, which had been estimated at 107 or 108 members [29,35]. An updated P. knowlesi genome is forthcoming, which resolves many of these gaps (Chappell et al., personal communication). 
(a)

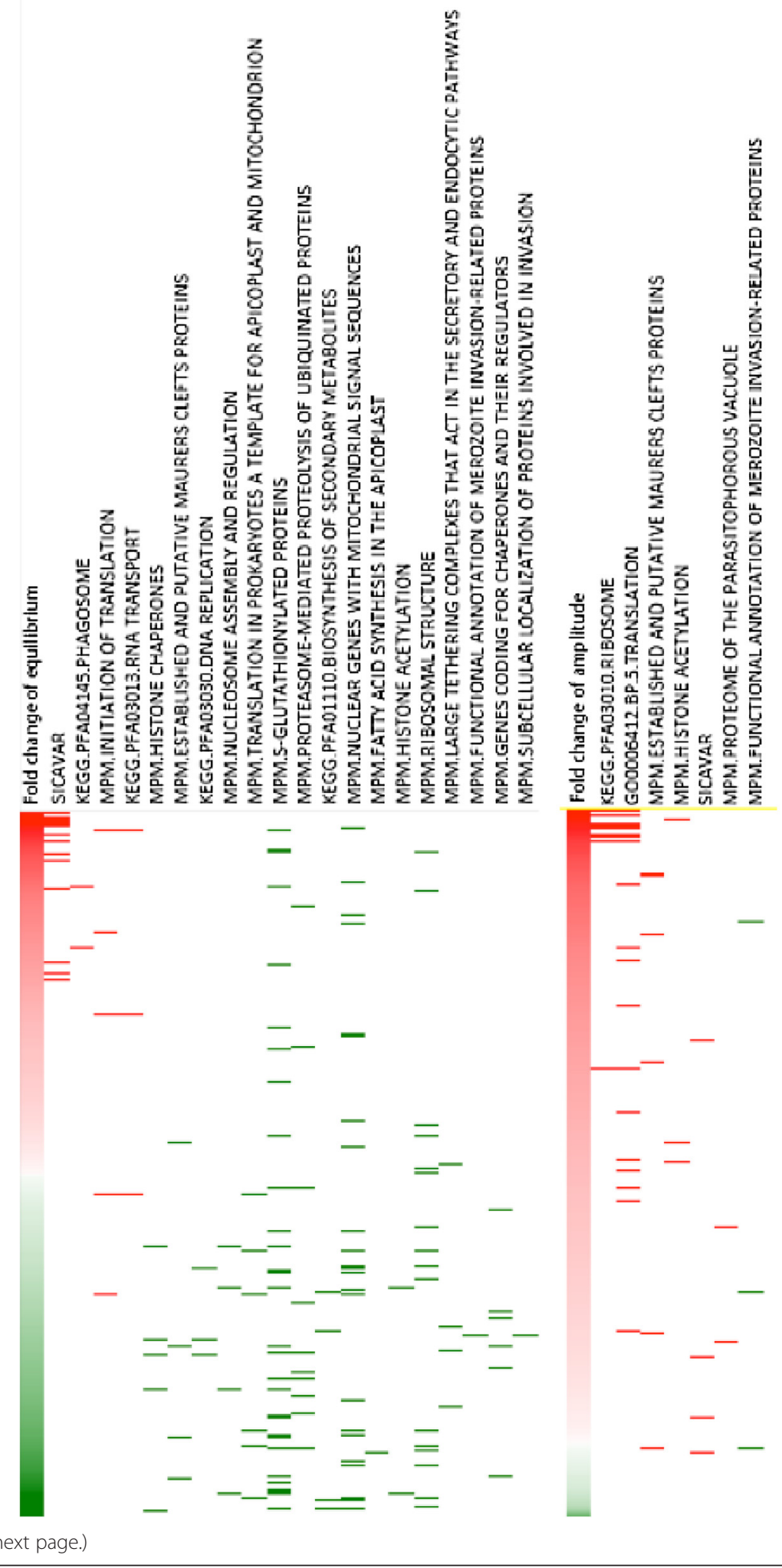


(See figure on previous page.)

Figure 4 Functional enrichment analysis of DE genes using GSEA software. (a) Significantly enriched functional groups for genes differentially expressed at equilibrium levels (FDR $<25 \%$, p value $<0.05$ ) (b) Enriched functional group of DE genes in amplitude (FDR $<25 \%$ ). Red color represents up regulated genes. Green color represents down regulated genes. The short lines indicate genes from the corresponding functional pathway that is shown in the header.

In contrast, only three out of $53(5.6 \%)$ of kir gene family members represented on the array were identified as DE over all TPs. Unlike the SICAvar genes, the kir genes were not up-regulated preferentially in the ring stages. Two kir genes were identified as being DE at TP1 (rings), four at TP2 (early trophozoites), and two at TP6 (schizonts). The remaining DE genes include 31 ribosomal genes $(4.5 \%)$ and various genes involved in either protein synthesis or processing.

\section{Genes differentially up-regulated ex vivo}

A number of other genes were found to be specifically up-regulated in the ex vivo cultured parasites (Additional file 6, Panels 1-6). The majority of these were more highly expressed in the earliest and latest stages of development (Figure 6). This subset includes genes involved in proteolysis (PKH_011450, proteasome subunit beta type gene) and radical-based catalysis ( $\mathrm{PKH} \_144460$, radical S-adenosyl methyltransferase protein gene), relevant for diverse metabolic reactions including biodegradation pathways. Interestingly, $\mathrm{PKH}_{-}$143910, the putative ApiAP2 transcription factor exhibited similar dynamics ex vivo and in vitro, but at a much higher level ex vivo, especially at earlier stages. Differential expression of genes involved in transcription in early stages has been documented before in P. falciparum [2,4]. Some unexpected transcripts were also detected such as the $P$. knowlesi homolog of pfg377 (PKH_146780), which encodes a protein that is specifically found on female gametocytes, and the PSOP12 homolog (PKH_101880) that encodes a

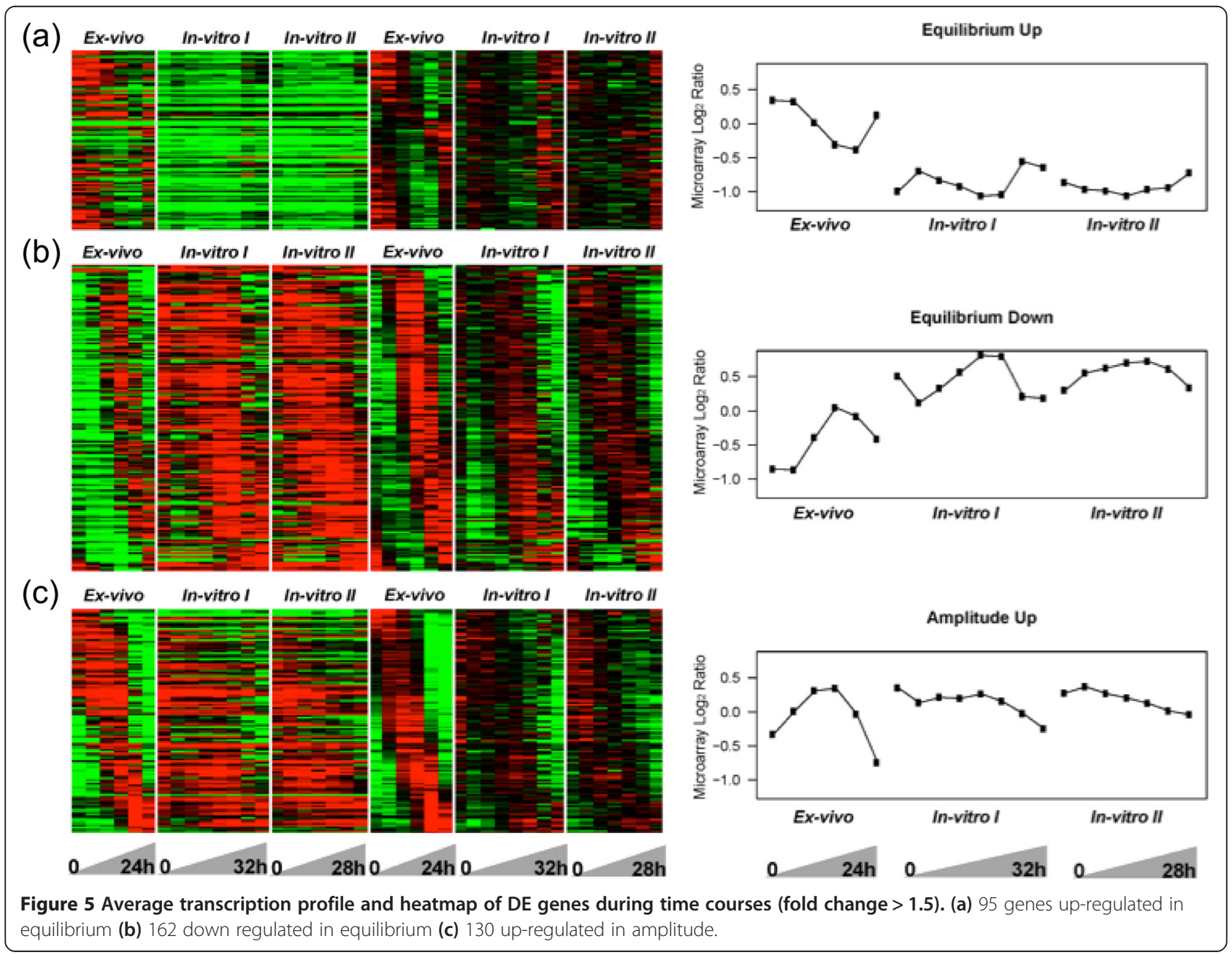




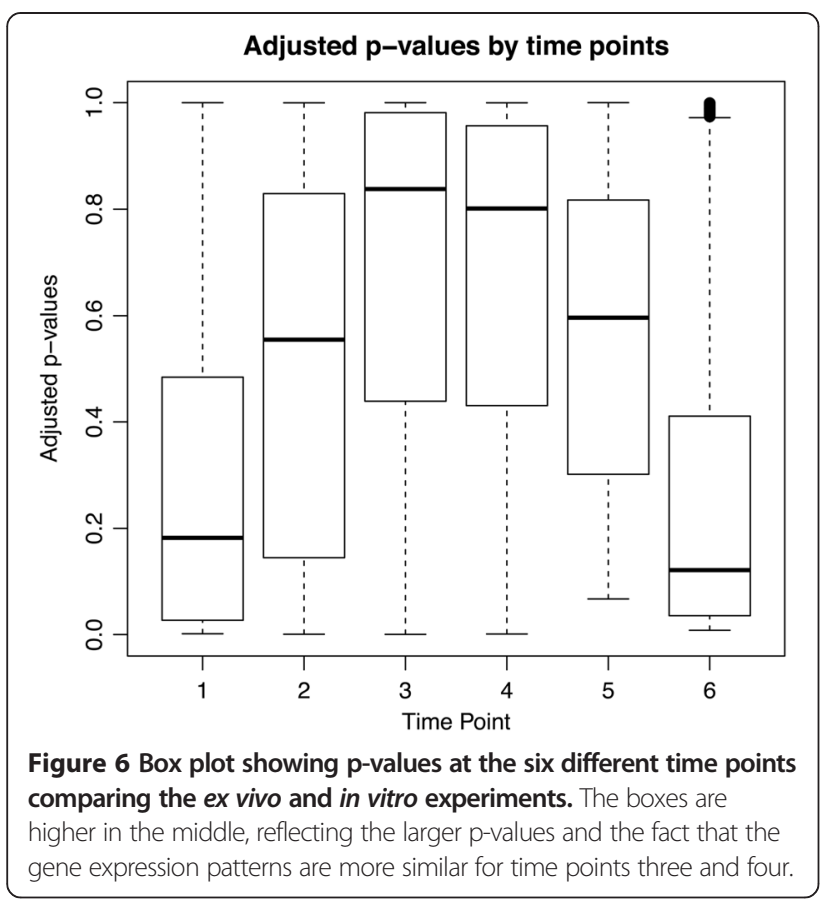

secreted protein at the ookinete stage. Gametocytes are often produced when the parasitaemia reaches high levels in rhesus macaques, and this could explain the detection of the pfg 377 homolog transcript. The ookinete protein could either have a separate function in the blood-stage parasites, or it is possible that the transcript is produced and maintained early, prior to its ultimate translation in the insect stage.

\section{Genes differentially up-regulated in vitro}

Only a few genes were found to be up-regulated in the in vitro compared to ex vivo-cultured parasites (Additional file 6, Panels 6-8). Most of these genes were expressed at higher levels in the later stages of the IDC. Several genes involved in metabolism and cell growth were significantly up-regulated in the in vitro cultures: e.g., porphobilinogen deaminase (PBGD) (PKH_130850) and protein tyrosine phosphatase (PKH_122120). The former is involved in the haem biosynthesis pathway, catalyzing porphobilinogen to uroporphyrinogen III (UROGEN III) (EC 2.5.1.61) [1,46]. The host PBGD, also called hydroxymethylbilane synthase, is most highly expressed in the spleen and bone marrow in humans based on tissue-specific ESTs (Additional file 7) [47]. Plasmodium parasites utilize host haem synthesis proteins in their erythrocytic life cycle in addition to their own de novo pathway [48], however the host PBGD is much more efficient [46]. It could be expected, then, to see parasite PBGD up-regulated when the host enzyme is dysregulated, as in a splenectomized host, or in in vitro culture. Conversely, if erythropoiesis is stimulated in the bone marrow during malaria infection, host PBGD may become up-regulated, decreasing the requirement of parasite de novo synthesis. Finally, one SICAvar gene (PKH_020570) was highly up-regulated in vitro in the schizonts (TP6), and less so in the ring-stage (TP1 and TP2) samples. While this could mean that this SICAvar gene or encoded protein has a unique role for the parasite's biology, the protein encoded by PKH_020570 was not identified in any blood-stage samples so far analysed by LC-MS/MS. It is also intriguing that this gene was also found to be up-regulated in P. knowlesi infected RBCs from a splenectomized animal (manuscript in preparation).

\section{Invasion and basic metabolic functions}

The basic invasion, growth and multiplication mechanisms of $P$. knowlesi parasites in vivo, ex-vivo and in vitro are similarly efficient. In each case, within a similar time frame ( 25 to 33 hours), the merozoites effectively enter $\mathrm{RBCs}$ and transform through the trophozoite and schizont stages of development to produce progeny merozoites. As anticipated, transcripts representing genes encoding proteins known to be involved in the invasion of RBCs were similarly detected, reflecting common IDCs for both the ex vivo and in vitro parasite samples; e.g. apical membrane antigen 1 [49], merozoite surface protein 3 [50,51], rhoptry associated proteins [52], and reticulocyte binding protein homologs [53,54] (see Additional file 6 Panels 14-22). Merozoite surface protein 1 (PKH_072850) did exhibit an oscillating pattern over time in one of the in vitro replicate experiments. All in all, while similarities were noted in many such comparisons, it remains possible that subtle differences and unnoticed temporal factors may make a big difference in the expression of various genes and the timely function of the encoded proteins. Small or subtle differences in transcript patterns detected may have an impact on protein expression and function, but overall the data are consistent with the fact that the ex vivo and in vitro-derived parasites efficiently invade and grow in rhesus RBCs. The differences in the timing of the bloodstage cycles, likely simply reflect the adjustment of the parasites from the synchronization procedures. It is also worth noting that similar transcript levels were found for genes with various basic metabolic functions. For example, 14-3-3 and actin transcripts were detected at similar levels (Additional file 6, Panels 25-26). Genes annotated as encoding blood-stage membrane proteins and integral membrane proteins were also examined, and with the exception of the SICAvar gene family (discussed below), found no significant differences (Additional file 6, Panels 23-24).

\section{Epigenetic regulation}

It is important to know whether Plasmodium genes that encode proteins involved in epigenetic regulation are 
differentially expressed in vivo compared to in vitro culture, especially given increasing knowledge of the importance of epigenetic regulation in Plasmodium [55-59], including the specific role of epigenetic regulation originally attributed to var gene expression in $P$. falciparum [60,61]. Overall, genes encoding histone and histone-modifying proteins exhibited similar levels of expression throughout the IDCs in the parasites grown ex vivo and in vitro. Interestingly, however, sir2 transcripts (PKH_122390) were more abundant in the late stages of ex vivo parasites compared to in vitro (Additional file 6, Panel 13). Further analysis is required to determine if this difference in expression is critical for the in vivo expression of SIR2 in relation to the specific regulation of the SICAvar gene family and the up-regulated expression of many SICAvar transcripts in the ring-stage parasites ex vivo.

\section{SICAvar gene expression is dramatically down-regulated in vitro}

The normalized intensity values of SICAvar genes were inputted into the Cluster program and visualized with TreeView (Figure 7). These data clearly show that the SICAvar gene family as a whole is down-regulated when the iRBCs are grown in vitro. In the ex vivo culture, the SICAvar gene IDs with the highest level of transcript expression demonstrated by the microarrays are PKH_050020 and PKH_072700/PKH_072710 (the latter two gene IDs represent a single SICAvar gene). These gene IDs were identified by LC-MS/MS for the $P$. knowlesi clone known as Pk1(A+), from which the $P$. knowlesi microarray oligonucleotides were based, but not for the $\mathrm{Pk} 1(\mathrm{~B}+) 1+$ clone [30]. Also detected at a high level was PKH_113950, which is highly expressed in the Pk1(B+)1+ clone [30]. However, a recent BLAST search revealed that the PKH_113950 oligonucleotide probe hits another SICAvar gene, PKH_020270, with $100 \%$ identity.

It was also determined if SICA proteins could be detected in the in vitro cultured parasites using LC-MS/ MS and found only one unique peptide corresponding to one SICA protein (PKH_030540); this contrasts to a high level of expression of multiple SICA proteins in established clones from rhesus macaque infections [30]. These findings are comparable to what is observed when P. knowlesi SICA[-] blood-stage parasites are grown in a splenectomized rhesus monkey, in which case neither functional full-length SICAvar transcripts nor SICA proteins are observed [30]. It has been known that the presence of the spleen is necessary for the expression and switching of SICA phenotypes in vivo [34], and that the spleen also has an apparent role in the expression of PfEMP1 encoded by the var genes and cytoadherence [62-64]. Whether only host splenic factors are critical for the expression of the SICAvar gene family or also other host influences is unknown. Similar questions remain valid with regards to the expression of the $P$. falciparum var genes in vivo. The stage-specific ex vivo microarray data are consistent with published expression library screening and standard RT-PCR experiments showing that many SICAvar genes are expressed as transcripts in the ring stage of development, without any evidence for the predominant transcription of any particular SICAvar transcript(s) [28-30,65].

One possible interpretation of these microarray data with regard to antigenic variation is that the default state for SICAvar genes in P. knowlesi is 'off', and that there are host cues that turn them 'on'. The spleen is one predicted source of these cues, since it has been shown that SICA protein expression at the surface of iRBCs is lost after passage in splenectomized hosts [34], and it has been shown quantitatively that the level of SICAvar transcripts detectable in splenectomized hosts is minimal in comparison with intact hosts. Moreover, full-length SICAvar transcripts are not detected in RNA extracted from SICA[-] parasites [30].

\section{Conclusions}

This stage-specific microarray analysis shows a marked difference in gene expression between $P$. knowlesi bloodstage parasites derived from a rhesus monkey infection and $P$. knowlesi parasites adapted for in vitro culture. Most strikingly, expression of the SICAvar variant antigen genes, which are related to the $P$. falciparum var genes and associated with increased virulence in vivo $[28,31,34]$ is up-regulated in the ex vivo cultures. Thus, in vitro cultures can provide a negative phenotype (in the "off state") for SICAvar gene expression in comparison with ex vivo samples (in the "on state"), similar to what has been demonstrated in splenectomized hosts $[30,34,63,66]$. Ex vivo cultures would therefore be important for unraveling the molecular mechanisms that control SICAvar gene expression and switching. Further investigations are required to evaluate what other genes may be implicated in the regulation of the SICAvar and potentially var gene family in the context of the in vivo host environment. Subsequent studies (Lapp, Chappel et al., personal communication) using RNA-Seq technologies will provide useful comparisons to expand upon the findings reported here.

Other clear examples of up-regulated and downregulated genes were also detected between the ex vivo and in vitro P. knowlesi samples, which should be taken into consideration when aiming to understand the parasite's natural programmed growth and development with the inherent influence of host factors in vivo. Critically, the data reported here bring emphasis to the fact that host-derived biological cues are important for Plasmodium gene expression. 


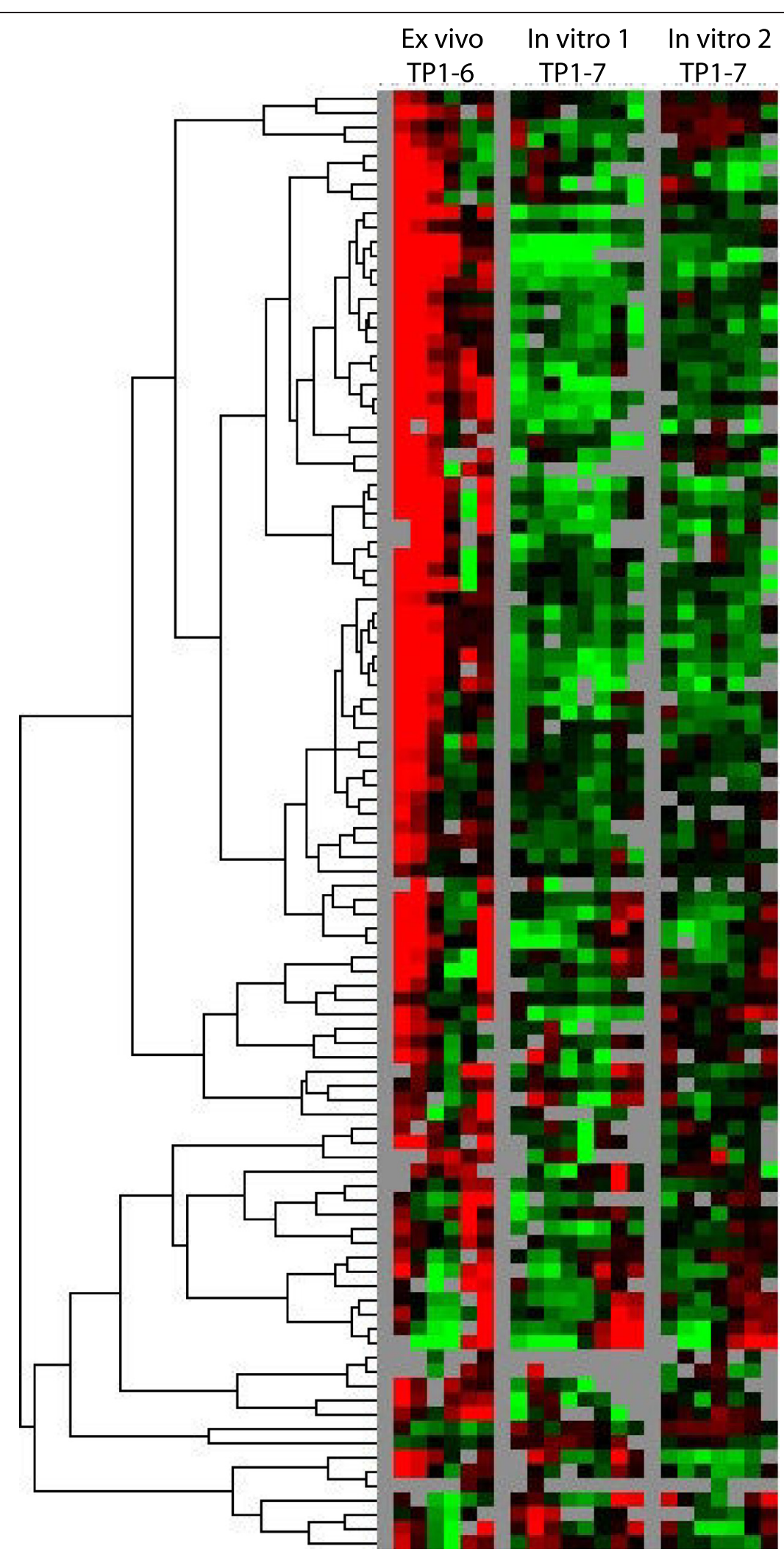

Figure 7 Heat map of all SICAvar genes in the ex vivo and two in vitro experiments, based on normalized intensity values. 


\section{Additional files}

Additional file 1: MA oligos. List of gene IDs and oligo sequences used in the analysis.

Additional file 2: Workflow. Data analysis workflow.

Additional file 3: PK4165genes_vivo_vitro. Data table of raw and centered intensities of the genes used for analysis.

Additional file 4: DE by stage. Differential expression of genes in ex vivo and in vitro by time point.

Additional file 5: Phase. Phase correlation between ex vivo and the two in vitro time courses.

Additional file 6: Panels showing comparative gene expression dynamics between ex vivo and in vitro time courses.

Additional file 7: ESTs of HMBS. Tissue-specific expression of HMBS in human.

\section{Competing interests}

The authors declare that they have no competing interests.

\section{Authors' contributions}

$S L$ designed and carried out the time course experiments, participated in the data analysis, and wrote the manuscript. SM performed the microarray experiments and statistical analyses. LZ performed statistical analyses and participated in the writing of the manuscript. HW performed statistical analyses. PP participated in designing the microarrays and critically reviewed the manuscript. ZB designed the microarrays, participated in strategic discussions and guided analyses. MG conceived the experiments, analyzed the data, and wrote the manuscript. All authors read and approved the final manuscript.

\section{Acknowledgements}

This project was funded in part by Federal funds from the US National Institute of Allergy and Infectious Diseases, National Institutes of Health, Department of Health and Human Services under grant \#R01-Al065961 and contract \# HHSN272201200031C, and supported in part by the Office of Research Infrastructure Programs /OD P51OD011132 (formerly National Center for Research Resources P51RR000165), as well as National Medical Research Council (Singapore) grant no. NMRC/1292/2011. We would also like to thank Kevin Lee for critical review and discussion of this work.

\section{Author details}

${ }^{1}$ Emory Vaccine Center, Yerkes National Primate Research Center, Emory University, Atlanta, GA, USA. ${ }^{2}$ School of Biological Sciences, Nanyang Technological University, Singapore, Singapore. ${ }^{3}$ Department of Biostatistics and Bioinformatics, Rollins School of Public Health, Emory University, Atlanta, GA, USA. ${ }^{4}$ Department of Medicine, Division of Infectious Diseases, Emory University, Atlanta, GA, USA.

Received: 14 November 2014 Accepted: 12 February 2015

Published online: 13 March 2015

\section{References}

1. Aurrecoechea C, Brestelli J, Brunk BP, Dommer J, Fischer S, Gajria B, et al PlasmoDB: a functional genomic database for malaria parasites. Nucleic Acids Res. 2009;37:D539-43.

2. Bozdech Z, Llinas M, Pulliam BL, Wong ED, Zhu J, DeRisi JL. The transcriptome of the intraerythrocytic developmental cycle of Plasmodium falciparum. PLOS Biol. 2003;1:E5.

3. Llinas M, Bozdech Z, Wong ED, Adai AT, DeRisi JL. Comparative whole genome transcriptome analysis of three Plasmodium falciparum strains. Nucleic Acids Res. 2006:34:1166-73.

4. Le Roch KG, Johnson JR, Florens L, Zhou Y, Santrosyan A, Grainger M, et al Global analysis of transcript and protein levels across the Plasmodium falciparum life cycle. Genome Res. 2004;14:2308-18.

5. Galinski MR, Meyer EV, Barnwell JW. Plasmodium vivax: modern strategies to study a persistent parasite's life cycle. Adv Parasitol. 2013;81:1-26.
6. Le Roch KG, Chung DW, Ponts N. Genomics and integrated systems biology in Plasmodium falciparum: a path to malaria control and eradication. Parasit Immunol. 2012;34:50-60.

7. Mwangi JM, Ranford-Cartwright LC. Genetic and genomic approaches for the discovery of parasite genes involved in antimalarial drug resistance. Parasitology. 2013;140:1455-67.

8. Trager W, Jensen JB. Human malaria parasites in continuous culture. Science. 1976:193:673-5.

9. Zeeman AM, der Wel AV, Kocken $\mathrm{CH}$. Ex vivo culture of Plasmodium vivax and Plasmodium cynomolgi and in vitro culture of Plasmodium knowlesi blood stages. Methods Mol Biol. 2013;923:35-49.

10. Moon RW, Hall J, Rangkuti F, Ho YS, Almond N, Mitchell GH, et al. Adaptation of the genetically tractable malaria pathogen Plasmodium knowlesi to continuous culture in human erythrocytes. Proc Natl Acad Sci U S A. 2013:110:531-6.

11. Golenda CF, Li J, Rosenberg R. Continuous in vitro propagation of the malaria parasite Plasmodium vivax. Proc Natl Acad Sci U S A. 1997;94:6786-91

12. Nguyen-Dinh P, Gardner AL, Campbell CC, Skinner JC, Collins WE. Cultivation in vitro of the vivax-type malaria parasite Plasmodium cynomolgi. Science. 1981:212:1146-8.

13. Noulin F, Borlon C, Van Den Abbeele J, D'Alessandro U, Erhart A. 1912-2012: a century of research on Plasmodium vivax in vitro culture. Trends Parasitol. 2013;29:286-94

14. Udomsangpetch R, Kaneko O, Chotivanich K, Sattabongkot J. Cultivation of Plasmodium vivax. Trends Parasitol. 2008;24:85-8.

15. Galinski MR, Barnwell JW. Plasmodium vivax: who cares? Malar J. 2008:7 Suppl 1:S9

16. Pologe LG, Ravetch JV. Large deletions result from breakage and healing of P. falciparum chromosomes. Cel. 1988:55:869-74.

17. LeRoux M, Lakshmanan V, Daily JP. Plasmodium falciparum biology: analysis of in vitro versus in vivo growth conditions. Trends Parasitol. 2009:25:474-81.

18. Peters JM, Fowler EV, Krause DR, Cheng Q, Gatton ML. Differential changes in Plasmodium falciparum var transcription during adaptation to culture. J Infect Dis. 2007;195:748-55.

19. Zhang $Q$, Zhang $Y$, Huang $Y$, Xue $X$, Yan $H$, Sun $X$, et al. From in vivo to in vitro: dynamic analysis of Plasmodium falciparum var gene expression patterns of patient isolates during adaptation to culture. PLoS One. 2011;6:e20591.

20. Fang J, Zhou H, Rathore D, Sullivan M, Su XZ, McCutchan TF. Ambient glucose concentration and gene expression in Plasmodium falciparum. Mo Biochem Parasitol. 2004;133:125-9.

21. Singh B, Kim Sung L, Matusop A, Radhakrishnan A, Shamsul SS, Cox-Singh J, et al. A large focus of naturally acquired Plasmodium knowlesi infections in human beings. Lancet. 2004;363:1017-24.

22. Galinski MR, Barnwell JW. Monkey malaria kills four humans. Trends Parasitol. 2009;25:200-4.

23. Hakimi H, Kawai S, Kawazu S. Molecular epidemiology of the emerging human malaria parasite "Plasmodium knowlesi". Trop Parasitol. 2014:4:20-4.

24. Bannister LH, Butcher GA, Dennis ED, Mitchell GH. Studies on the structure and invasive behaviour of merozoites of Plasmodium knowlesi. Trans R Soc Trop Med Hyg. 1975;69:5.

25. Aikawa M, Miller LH, Johnson J, Rabbege J. Erythrocyte entry by malaria parasites. A moving junction between erythrocyte and parasite. J Cell Biol. 1978;77:72-82

26. Brown KN, Brown IN. Immunity to malaria: antigenic variation in chronic infections of Plasmodium knowlesi. Nature. 1965:208:1286-8.

27. Howard RJ, Barnwell JW, Kao V. Antigenic variation of Plasmodium knowlesi malaria: identification of the variant antigen on infected erythrocytes. Proc Natl Acad Sci U S A 1983:80:4129-33.

28. Al-Khedery B, Barnwell JW, Galinski MR. Antigenic variation in malaria: a 3' genomic alteration associated with the expression of a $P$. knowlesi variant antigen. Mol Cell. 1999;3:131-41.

29. Corredor V, Meyer EV Lapp S, Corredor-Medina C, Huber CS, Evans AG, et al. A SICAvar switching event in Plasmodium knowlesi is associated with the DNA rearrangement of conserved 3' non-coding sequences. Mol Biochem Parasitol. 2004;138:37-49.

30. Lapp SA, Korir-Morrison C, Jiang J, Bai Y, Corredor V, Galinski MR. Spleen-dependent regulation of antigenic variation in malaria parasites: Plasmodium knowlesi SICAvar expression profiles in splenic and asplenic hosts. PLoS One 2013:8:e78014 
31. Korir CC, Galinski MR. Proteomic studies of Plasmodium knowlesi SICA variant antigens demonstrate their relationship with $P$. falciparum EMP1. Infect Genet Evol. 2006;6:75-9.

32. Frech $\mathrm{C}$, Chen N. Variant surface antigens of malaria parasites: functional and evolutionary insights from comparative gene family classification and analysis. BMC Genomics. 2013;14:427.

33. Kyes S, Pinches R, Newbold C. A simple RNA analysis method shows var and rif multigene family expression patterns in Plasmodium falciparum. Mol Biochem Parasitol. 2000;105:311-5.

34. Barnwell JW, Howard RJ, Coon HG, Miller LH. Splenic requirement for antigenic variation and expression of the variant antigen on the erythrocyte membrane in cloned Plasmodium knowlesi malaria. Infect Immun. 1983:40:985-94.

35. Pain A, Bohme $U$, Berry AE, Mungall K, Finn RD, Jackson AP, et al. The genome of the simian and human malaria parasite Plasmodium knowlesi. Nature. 2008;455:799-803.

36. Lambros C, Vanderberg JP. Synchronization of Plasmodium falciparum erythrocytic stages in culture. J Parasitol. 1979;65:418-20.

37. Peng J, Elias JE, Thoreen CC, Licklider LJ, Gygi SP. Evaluation of multidimensional chromatography coupled with tandem mass spectrometry (LC/LC-MS/MS) for large-scale protein analysis: the yeast proteome. J Proteome Res. 2003;2:43-50.

38. Mok S, Imwong M, Mackinnon MJ, Sim J, Ramadoss R, Yi P, et al. Artemisinin resistance in Plasmodium falciparum is associated with an altered temporal pattern of transcription. BMC Genomics. 2011:12:391.

39. Subramanian A, Tamayo P, Mootha VK, Mukherjee S, Ebert BL, Gillette MA, et al. Gene set enrichment analysis: a knowledge-based approach for interpreting genome-wide expression profiles. Proc Natl Acad Sci U S A. 2005;102:15545-50.

40. Tachibana SI, Sullivan SA, Kawai S, Nakamura S, Kim HR, Goto N, et al. Plasmodium cynomolgi genome sequences provide insight into Plasmodium vivax and the monkey malaria clade. Nat Genet. 2012;44:1051-5.

41. Smyth GK. limma: Linear Models for Microarray Data. In: Gentleman R, Carey V, Huber W, Irizarry R, Dudoit S, editors. Bioinformatics and Computational Biology Solutions Using R and Bioconductor. New York: Springer; 2005. p. 397-420. Statistics for Biology and Health.

42. Coffey N, Hinde J. Analyzing time-course microarray data using functional data analysis - a review. Stat Appl Genet Mol Biol. 2011;10:10-32.

43. Kanehisa M, Goto S, Kawashima S, Okuno Y, Hattori M. The KEGG resource for deciphering the genome. Nucleic Acids Res. 2004;32:D277-80.

44. Malaria Parasite Metabolic Pathways. [http://sites.huji.ac.il/malaria/]

45. Ashburner M, Ball CA, Blake JA, Botstein D, Butler H, Cherry JM, et al. Gene ontology: tool for the unification of biology. The Gene Ontology Consortium. Nat Genet. 2000;25:25-9.

46. Nagaraj VA, Arumugam R, Gopalakrishnan B, Jyothsna YS, Rangarajan PN, Padmanaban G. Unique properties of Plasmodium falciparum porphobilinogen deaminase. J Biol Chem. 2008;283:437-44.

47. Liu X, Yu X, Zack DJ, Zhu H, Qian J. TiGER: a database for tissue-specific gene expression and regulation. BMC Bioinformatics. 2008:9:271.

48. Nagaraj VA, Sundaram B, Varadarajan NM, Subramani PA, Kalappa DM, Ghosh SK, et al. Malaria parasite-synthesized heme is essential in the mosquito and liver stages and complements host heme in the blood stages of infection. PLoS Pathog. 2013;9:e1003522.

49. Thomas AW, Narum D, Waters AP, Trape JF, Rogier C, Goncalves A, et al Aspects of immunity for the AMA-1 family of molecules in humans and non-human primates malarias. Mem Inst Oswaldo Cruz. 1994;89 Suppl 2:67-70.

50. Hudson DE, Miller LH, Richards RL, David PH, Alving CR, Gitler C. The malaria merozoite surface: a 140,000 m.w. protein antigenically unrelated to other surface components on Plasmodium knowlesi merozoites. J Immunol. 1983;130:2886-90.

51. Jiang J, Barnwell JW, Meyer EV, Galinski MR. Plasmodium vivax merozoite surface protein-3 (PVMSP3): expression of an 11 member multigene family in blood-stage parasites. PLoS One. 2013;8:e63888.

52. Counihan NA, Kalanon M, Coppel RL, de Koning-Ward TF. Plasmodium rhoptry proteins: why order is important. Trends Parasitol. 2013;29:228-36.

53. Meyer EV, Semenya AA, Okenu DM, Dluzewski AR, Bannister LH, Barnwell $J W$, et al. The reticulocyte binding-like proteins of $P$. knowlesi locate to the micronemes of merozoites and define two new members of this invasion ligand family. Mol Biochem Parasitol. 2009;165:111-21.
54. Semenya AA, Tran TM, Meyer EV, Barnwell JW, Galinski MR. Two functional reticulocyte binding-like (RBL) invasion ligands of zoonotic Plasmodium knowlesi exhibit differential adhesion to monkey and human erythrocytes. Malar J. 2012:11:228

55. Duffy MF, Selvarajah SA, Josling GA, Petter M. Epigenetic regulation of the Plasmodium falciparum genome. Brief Funct Genomics. 2014;13:203-16.

56. Gupta AP, Chin WH, Zhu L, Mok S, Luah YH, Lim EH, et al. Dynamic epigenetic regulation of gene expression during the life cycle of malaria parasite Plasmodium falciparum. PLoS Pathog. 2013;9:e1003170.

57. Cui L, Miao J. Chromatin-mediated epigenetic regulation in the malaria parasite Plasmodium falciparum. Eukaryot Cell. 2010;9:1138-49.

58. Hoeijmakers WA, Salcedo-Amaya AM, Smits AH, Francoijs KJ, Treeck M, Gilberger TW, et al. H2A.Z/H2B.Z double-variant nucleosomes inhabit the AT-rich promoter regions of the Plasmodium falciparum genome. Mol Microbiol. 2013;87:1061-73.

59. Cortes A, Carret C, Kaneko O, Yim Lim BY, Ivens A, Holder AA. Epigenetic silencing of Plasmodium falciparum genes linked to erythrocyte invasion. PLOS Pathog. 2007:3:e107.

60. Voss TS, Tonkin CJ, Marty AJ, Thompson JK, Healer J, Crabb BS, et al. Alterations in local chromatin environment are involved in silencing and activation of subtelomeric var genes in Plasmodium falciparum. Mol Microbiol. 2007:66:139-50.

61. Petter M, Lee CC, Byrne TJ, Boysen KE, Volz J, Ralph SA, et al. Expression of P. falciparum var genes involves exchange of the histone variant H2A.Z at the promoter. PLoS Pathog. 2011;7:e1001292.

62. Hommel M, David PH, Oligino LD. Surface alterations of erythrocytes in Plasmodium falciparum malaria. Antigenic variation, antigenic diversity, and the role of the spleen. J Exp Med. 1983;157:1137-48.

63. Munasinghe A, lleperuma M, Premawansa G, Handunnetti S, Premawansa S. Spleen modulation of cytoadherence properties of Plasmodium falciparum. Scand J Infect Dis. 2009:41:538-9.

64. Pongponratn E, Viriyavejakul P, Wilairatana P, Ferguson D, Chaisri U, Turner $G$, et al. Absence of knobs on parasitized red blood cells in a splenectomized patient in fatal falciparum malaria. Southeast Asian J Trop Med Public Health. 2000;31:829-35.

65. Galinski MR, Corredor V. Variant antigen expression in malaria infections: posttranscriptional gene silencing, virulence and severe pathology. Mol Biochem Parasitol. 2004;134:17-25.

66. Barnwell JW, Howard RJ, Miller LH. Altered expression of Plasmodium knowlesi variant antigen on the erythrocyte membrane in splenectomized rhesus monkeys. J Immunol. 1982;128:224-6.

\section{Submit your next manuscript to BioMed Central and take full advantage of:}

- Convenient online submission

- Thorough peer review

- No space constraints or color figure charges

- Immediate publication on acceptance

- Inclusion in PubMed, CAS, Scopus and Google Scholar

- Research which is freely available for redistribution 